\title{
A review of late-stage tungsten fuzz growth
}

\author{
Jacob A. R. Wright ${ }^{1}$ (1) \\ Received: 4 October 2021 / Revised: 19 November 2021 / Accepted: 20 November 2021 / Published online: 12 January 2022 \\ (c) The Author(s) 2022
}

\begin{abstract}
Tungsten will be used as the plasma-facing divertor material in the International Thermonuclear Experimental Reactor (ITER) fusion reactor. Under high temperatures and high ion fluxes, a 'fuzz' nanostructure forms on the tungsten surface with dramatically different properties and could contaminate the plasma. Although simulations and experimental observations have provided understanding of the initial fuzz formation process, there is debate over whether tungsten or helium migration is rate-limiting during late-stage growth, and the mechanisms by which tungsten and helium migrations occur. Here, the proposed mechanisms are considered in turn. It is concluded that tungsten migration occurs by adatom diffusion along the fuzz surface. Continual helium migration through the porous fuzz to the tungsten bulk is also required for fuzz growth, for continued bubble growth and rupture. Helium likely migrates due to ballistic penetration, although diffusion may contribute. It is difficult to determine the limiting process, which may switch from helium penetration to tungsten adatom diffusion above a threshold flux. Areas for further research to clarify the mechanisms are then considered. A greater understanding of the fuzz formation mechanism is key to the successful design of plasma-facing tungsten components, and may have applications in forming porous tungsten catalysts.
\end{abstract}

Keywords Tungsten $\cdot$ Tungsten fuzz $\cdot$ Plasma-material interactions $\cdot$ Fusion reactors

\section{Introduction}

Fusion reactors can provide a safe, clean power supply using readily available raw materials. However, developing materials that are able to withstand the extreme conditions is key to making fusion viable. The International Thermonuclear Experimental Reactor (ITER) will be a tokamak reactor, which confines the plasma within a torus, as shown in Fig. 1 [1].

The divertor is at the torus base, where waste material exits. It must protect surrounding components from extreme heat, impinging ions, and neutrons [2]. In ITER, the divertor will be made of tungsten, selected for its high melting point, high thermal conductivity, and low sputter yields (the number of tungsten ions ejected from the material per incident ion) [3].

Both hydrogen and helium ions impinge upon the divertor, but this review focusses only on the microstructural

Jacob A. R. Wright

jarw2@cam.ac.uk

1 Department of Materials Science and Metallurgy, University of Cambridge, Cambridge CB3 OFS, UK change named fuzz, caused by helium ions $\left(\mathrm{He}^{+}\right)$. Fuzz is a porous layer that forms on top of the tungsten bulk, shown in Fig. 2 [4]. The layer can grow beyond several micrometres thick and consists of individual tendrils with diameters of $10-50 \mathrm{~nm}$ [5].

The conditions for fuzz formation, which is expected during ITER operation [6], are discussed in Sect. 2.3. The fuzz layer could reduce the divertor's thermal conductivity [7] and contaminate the plasma [8]. Understanding the fuzz formation mechanism will be key to preventing these problems, and could allow fuzz to be used for beneficial purposes, such as catalysing water splitting [9] or organic compound breakdown [10].

Due to the extreme conditions of fuzz formation, mechanisms are commonly investigated by simulation studies. The available experimental data can then be used to validate predictions from these studies. Simulations are effective at modelling the first stages of fuzz formation, where helium ions impact on perfect tungsten bulk material, and the subsequent motion of helium within the bulk. This has led to a good understanding of the bubble growth and rupture mechanism [11], discussed in Sect. 2.2. However, it has not been possible for simulations to reproduce a complete fuzz structure [8] and outstanding questions remain. 


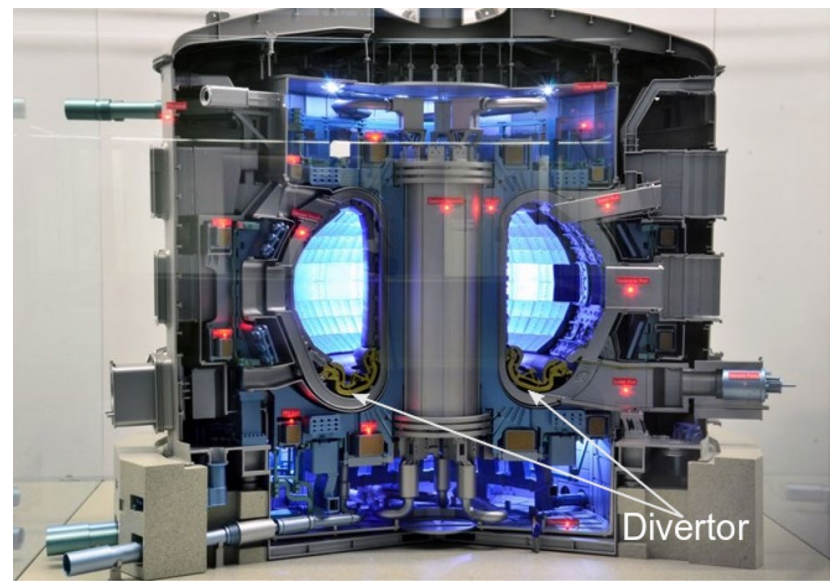

Fig. 1 ITER scale model. Arrows indicate the tungsten divertor at the base of the torus. Reproduced with permission from Ref. [1]. Copyright 2013. ITER.org

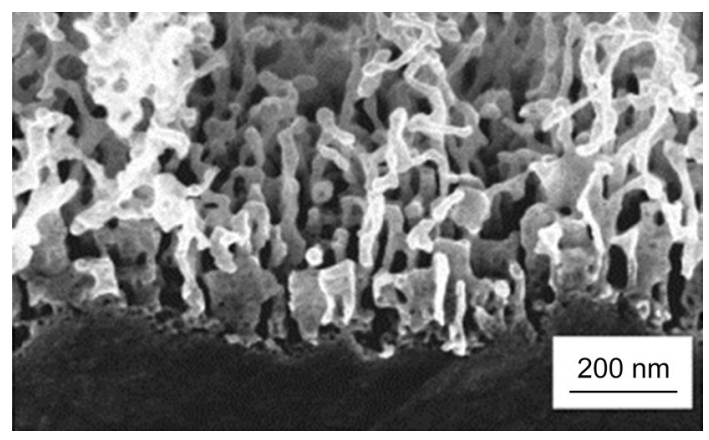

Fig. 2 SEM image showing initial fuzz formation on tungsten bulk. Reproduced with permission from Ref. [4]. Copyright 2009. International Atomic Energy Agency

The current consensus on fuzz formation conditions and mechanism is presented in Sect. 2. Sections 3 and 4 discuss mechanisms proposed to explain tungsten and helium migration, respectively. Section 5 examines which of these is ratelimiting. Section 6 presents the conclusions and identifies areas for further study.

For clarity, in this review, the overall microstructure change is described as fuzz, which consists of individual tendrils.

\section{Current consensus}

This section introduces the accepted mechanism of bubble growth and rupture, and the outstanding questions of fuzz formation. The conditions, and the variation of growth with these conditions, are also presented. The different theories presented in Sects. 3 and 4 can then be evaluated on how well they explain the experimental evidence.

\subsection{Bubble growth and rupture}

It is accepted that He ions implant into the $\mathrm{W}$ surface, leading to the nucleation of He bubbles underneath the surface. These bubbles then grow and rupture; subsequently fuzz forms. Continued bubble rupture leads to extremely highspecific surface areas $[12,13]$, highlighting the potential of W fuzz-based catalysts. Different mechanisms have been proposed to explain how this bubble growth leads to fuzz formation. The mechanism of bubble growth and rupture is summarised below and in Fig. 3 [14].

1. A He ion implants within the $\mathrm{W}$ bulk, where there is low He solubility. This leaves a He atom that diffuses until it desorbs from the surface [15] or encounters another $\mathrm{He}$ atom, forming a mobile cluster of He atoms. These
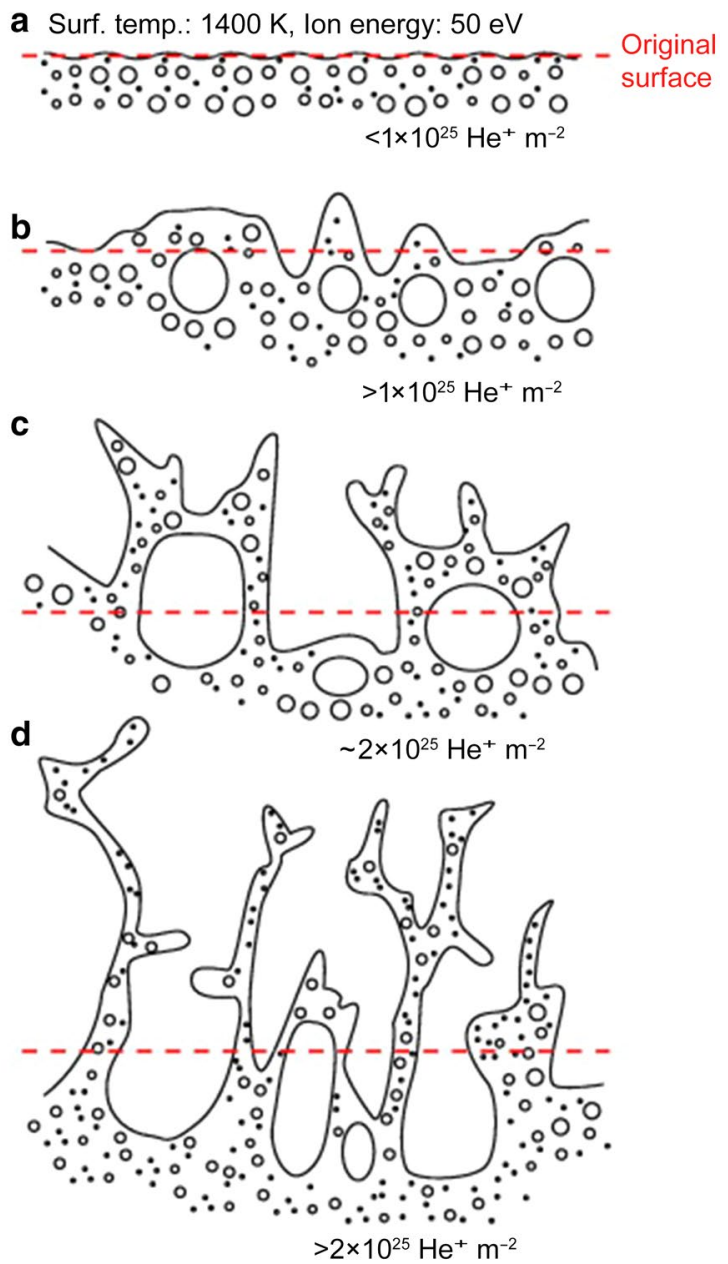

Fig. 3 Schematics of initial fuzz growth from TEM images, with labelled fluences. The red dotted line marks the initial surface. a shows the initial formation of many small bubbles. $\mathbf{b}$ and $\mathbf{c}$ illustrate the growth of large bubbles, causing outward surface growth. $\mathbf{d}$ shows the sharp protrusions formed by bubble rupture. Reproduced with permission from Ref. [14]. Copyright 2011. Elsevier 
clusters can grow by absorbing other clusters or lone $\mathrm{He}$ atoms [11].

2. Once large enough, these clusters can eject a $\mathrm{W}$ atom into an interstitial site, leaving the He cluster bound to the lattice vacancy, a Frenkel pair. The cluster could also combine with a vacancy already present in the lattice instead [16]. Immobile clusters bound to vacancies are called bubbles [11].

3. These bubbles absorb more He and grow by trap mutation. This is where a $\mathrm{VHe}_{n}$ bubble (consisting of one vacancy, $\mathrm{V}$ ) grows to $\mathrm{V}_{2} \mathrm{He}_{n}$ and produces another interstitial $\mathrm{W}$ atom, which remains bound to the bubble surface.

4. Once the number of interstitials is high enough, they form a dislocation loop (the loop surrounds a disc of $\mathrm{W}$ interstitials forming an extra plane of atoms in the lattice) [11]. The loop is no longer bound to the bubble, and is free to travel to the surface and annihilate. This is loop punching, which causes the surface to grow outwards [16]; the disc of interstitials is now a group of adatoms - atoms on the $\mathrm{W}$ surface.

5. As loop punching continues, the distance from the bubble to the initial surface decreases, causing the bubble to rupture. This leaves protrusions and pits which form the basis of the fuzz tendrils [14], illustrated in Fig. 3. Simulations can reproduce this, showing that protrusions are caused by bubble bursting [17-19]. Transmission electron microscopy (TEM) images have shown that in addition to the large bubbles that rupture, there are many small bubbles within the bulk and the individual tendrils [14]. The stresses within the large bubbles, and from the surrounding smaller bubbles, likely drive bubble rupture [20].

\subsection{Conditions}

Table 1 shows the conditions for fuzz formation. The observed incubation fluence (the minimum fluence before fuzz growth) is required for bubbles to form [21] which are required for fuzz growth [22].

At high temperatures, due to the low thermal conductivity of the fuzz [7, 23], tendrils heat up and reintegrate back into the bulk. Irradiation by impurities or high-energy He ions ( $>120 \mathrm{eV}$ ) can lead to sputtering, the emission of $\mathrm{W}$ atoms from the sample, which erodes surface features [24]. This erosion competes with fuzz growth and the fuzz reaches a steadystate thickness, where these effects balance [25]. Increasing

Table 1 Observed conditions of fuzz formation. Data from Ref. [6]

\begin{tabular}{lll}
\hline Temperature & Minimum $\mathrm{He}^{+}$energy & Minimum $\mathrm{He}^{+}$fluence \\
\hline $1000-2000 \mathrm{~K}$ & $\sim 20 \mathrm{eV}$ & $10^{24} \mathrm{~m}^{-2}$ \\
\hline
\end{tabular}

ion energies reduce this thickness significantly. At even higher ion energies $(>10 \mathrm{keV})$, larger fuzz-like structures form again [11]. However, the ITER divertor will be subject to energies in the range $0-100 \mathrm{eV}$, which we focus upon.

The minimum energy occurs because ions below $\sim 20 \mathrm{eV}$ are more likely to be reflected by the surface [11] so cannot easily form bubbles. For example, $12 \mathrm{eV}$ ions only formed fuzz at extremely high fluences $\left(>10^{27} \mathrm{~m}^{-2}\right)$ [26], due to the high reflectance. Thus, $20 \mathrm{eV}$ is taken as a practical minimum energy.

It has been observed that the growth rate decreases over time, and is proportional to the square root of the exposure time. It was therefore concluded that diffusion through the fuzz is rate-limiting [27]. Thicker fuzz leads to greater diffusion distances, slowing the growth rate. Since this conclusion, growth has been analysed using the one-dimensional diffusion length (from Fick's laws) [28]:

$x=\sqrt{2 D t}$,

where $x$ is fuzz thickness and $D$ is an effective diffusion coefficient. Since He flux, $\Gamma$, is typically constant, fluence, $\Phi=\Gamma t$. There is a square-root dependence on fluence too, as fluence is proportional to time. Substituting for $t$ and including the incubation fluence, $\Phi_{0}$, gives the relationship between fuzz thickness and He fluence:

$x=\sqrt{\frac{2 D}{\Gamma}\left(\Phi-\Phi_{0}\right)}$.

The incubation time is related by $\Phi_{0}=\Gamma t_{0}$.

However, which species is responsible for diffusion was not clear. A logarithmic model has also been proposed to explain the decreasing growth rate, based on the penetration of He ions. These areas of debate are discussed in Sect. 5. Until then, we compare mechanisms to the currently accepted $\sqrt{t}$ dependence.

This leaves interesting questions for the proposed mechanisms to answer:

- Is $\mathrm{W}$ migration, He migration, or both responsible for fuzz formation?

- If a He supply is required, how does He move through the thickening fuzz layer?

- Is $\mathrm{W}$ or He migration rate-limiting?

\section{Tungsten migration}

This section explores the proposed mechanisms for $\mathrm{W}$ migration during late-stage fuzz formation. The theory, evidence supporting it, and evidence against it is examined in turn for each model. 


\subsection{Electrostatic growth}

This theory proposes that fuzz could be formed from metal whiskers growing on the W surface. Protrusions would induce large electric fields, favouring whisker growth [29]. The interaction with the electric field from the plasma present could accelerate this.

However, two studies have shown that electric field variation has no effect on fuzz growth [30,31], so this mechanism has been rejected.

\subsection{Viscoelastic flow}

This was proposed by Krasheninnikov [32], suggesting that bubbles within the growing tendrils exert stresses which cause plastic deformation-creep-where $\mathrm{W}$ flows up tendrils causing growth, shown in Fig. 4. The bubbles in tendrils experience a drift force attracting them to free surfaces, which causes them to push upwards to the top of the tendrils [11].

Krasheninnikov et al. [32] concluded that whichever of the He or W supply was slower would lead to the $\sqrt{t}$ dependence. At a constant He flux, the growth rate decreases over time [33] (as thickness is proportional to $\sqrt{t}$ ); this was interpreted as evidence that $\mathrm{W}$ supply was limiting. This model was also able to reproduce the $\sqrt{t}$ dependence. Subsequent molecular dynamics (MD) calculations have shown that large amounts of He reduce the yield strength of W [34], supporting the proposal of plastic deformation.

However, there is opposing evidence. It has been found that $\mathrm{He}$ concentrations do not reach the necessary level to cause W yield [35]. Also, a recent experiment using ${ }^{3} \mathrm{He}$ and ${ }^{4} \mathrm{He}$ isotopes [36] found that bubbles continually rupture during growth. Only ${ }^{4} \mathrm{He}$ was released for the final part of plasma exposure and only ${ }^{4} \mathrm{He}$ was found in the bulk or fuzz. Thus, all ${ }^{3} \mathrm{He}$ was released, by bubble rupture, and ${ }^{4} \mathrm{He}$ was able to move through the fuzz layer. This theory does not consider the continual rupture of bubbles. In addition, the assumption that W supply is limiting is premature. Even at constant flux of He ions, the transport of He through the fuzz layer to the bulk could be rate-limiting, as considered later.

\subsection{Adatom diffusion}

In this theory, $\mathrm{He}^{+}$ion bombardment leads to the formation of adatoms, i.e. atoms on the $\mathrm{W}$ surface. These diffuse along the sides of tendrils, to the tips. Whilst this appears unphysical, explanations for this phenomenon are given in the next paragraph. This mechanism is diffusive, thus explaining the $\sqrt{t}$ dependence of growth. An MD study [37] calculated the effective adatom diffusion coefficients, which were similar to those obtained experimentally [27].

It has been proposed that adatoms diffuse to tendril tips due to stresses from He bubbles, which lower the chemical potential in the high-curvature tip regions [38]. This is supported by recent work finding that stresses from He bubbles can drive $\mathrm{W}$ adatom diffusion [12]. Additionally, an experimental study [22] found that fuzz formed when a sample was irradiated with $\mathrm{He}^{+}$, then held above $1273 \mathrm{~K}$ without any further irradiation. Thus, if He bubbles pre-exist, no more $\mathrm{He}$ is needed to form fuzz initially. Fuzz even formed on very thin regions of this sample without any He bubbles present. This was attributed to $\mathrm{W}$ adatoms diffusing driven by surface tension. Under further He irradiation, bubbles formed in the thin regions, increasing the growth rate. The rate increase provides evidence that bubbles enhance surface diffusion of adatoms. In a thick sample (relevant to fusion reactors), He bubbles are required for fuzz initiation; this could be to drive adatom diffusion. Finally, annealing fuzz [23] leads to He release and subsequent destruction of the fuzz nanostructure; without the stresses from He bubbles, the metastable fuzz disappears when heated. a

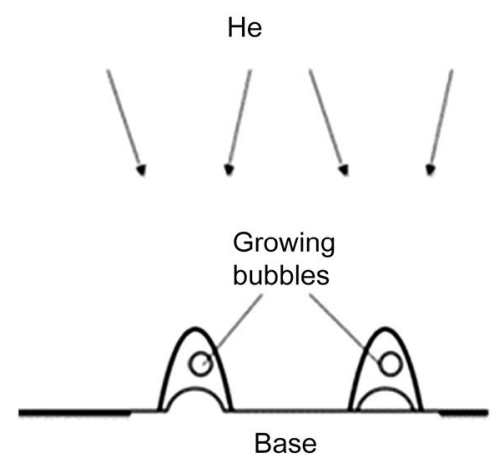

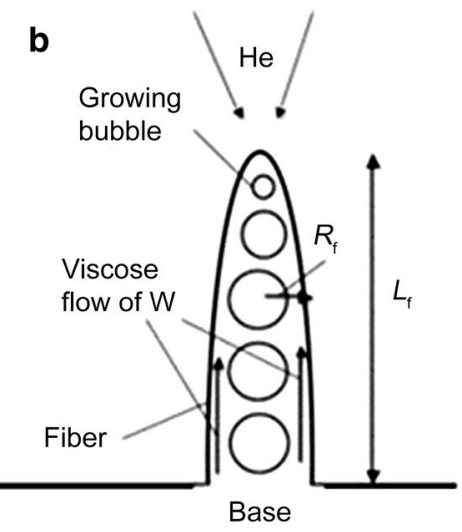

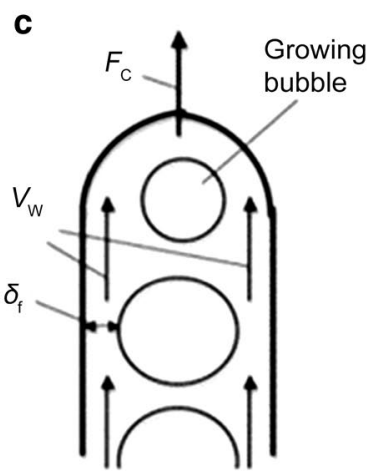

Fig. 4 Side-on tendril views explaining the viscoelastic mechanism. a illustrates the initial tendril, $\mathbf{b}$ and $\mathbf{c}$ show the viscous flow of W to the tip due to the bubble pressure. Reproduced with permission from Ref. [32]. Copyright 2011. IOP Publishing 
Fiflis et al. [30] replaced $\mathrm{He}^{+}$ions with $\mathrm{Ne}^{+}$which did not lead to fuzz. Both should produce adatoms by bombardment, so both should form fuzz. The authors concluded that adatoms were not responsible for fuzz growth. However, the different penetrations of $\mathrm{He}^{+}$and $\mathrm{Ne}^{+}$mean the $\mathrm{Ne}^{+}$will not lead to bubble formation [39] which we have seen is necessary for adatom diffusion. Thus, their conclusion was premature.

Recent studies [40-42] have deposited W atoms on the sides of growing tendrils, like those in Fig. 3d. They found that this increases the growth rate significantly, such that there is no longer a $\sqrt{t}$ dependence. This is evidence that adatom diffusion is responsible for growth. However, this does not establish whether adatom migration or adatom generation is limiting during normal growth because both processes are enhanced by deposition. Adatom migration is increased as adatoms deposited on the sides of tendrils have a shorter distance to the tip than those generated at the base. It was also observed that the tendril widths remain constant even with extra $\mathrm{W}$ deposition on the sides, further supporting adatom diffusion along tendril surfaces to the tip.

Hammond [11] argues that adatoms alone cannot account for fuzz growth. Their migration is too slow, and there is little uphill diffusion, where an adatom diffuses to an island of many adatoms. This means the island cannot grow to form the initial protrusions. However, adatoms do not need to be responsible for this; bubble rupture can form protrusions, as seen in Fig. 3, and adatoms can then diffuse to the protrusion tips [43].

The original theory suggested adatoms would form on the side of tendrils due to He bombardment. However, experimental studies with W isotope films [36] on W, thin Mo films on W (Mo forms similar fuzz) [30, 44, 45], and thin W films on Mo [45] have found that the surface and bulk materials mix in fuzz formation. Thus, bubble growth, rupture and loop punching (discussed in Sect. 2.2) are responsible for forming adatoms [46], bringing material up from the bulk. If adatoms formed immediately due to He bombardment, mixing would not occur as only surface material would form adatoms. Instead, He bubbles are responsible for adatom formation, which can diffuse over the surface with a net upward flux to the tip, as detailed above. The continual bubble rupture, evidence for which is discussed in Sect. 2.2, is responsible for generating adatoms throughout the process.

This explains why the supply of new material is from the bulk. It was also observed that there is greater concentration of bulk material at the edge of tendrils $[36,44]$. This supports adatom migration over viscoelastic flow. If flow occurred within the tendrils, shown in Fig. 4, a large concentration of atoms from the bulk would not be seen at the edges of tendrils. During late-stage growth, bubbles within the fuzz could also lead to adatom formation on the side of tendrils by the same mechanism [8].

To summarise:

- Unlike the original theory suggested, adatoms are not formed directly due to He bombardment.

- Adatoms are created on the $\mathrm{W}$ surface by bubble growth and loop punching.

- Bubble rupture then forms a crater with protrusions.

- Adatoms diffuse to the tips of these protrusions (the precursors to tendrils) along the surfaces causing growth.

- Adatoms continue to be generated by loop punching of He bubbles during late-stage growth.

This means a continuous supply of He through the fuzz layer to the $\mathrm{W}$ bulk is necessary for bubbles to continue to produce adatoms. The rate-limiting process is either adatom migration (diffusion along tendril surfaces) or generation. If generation is rate-limiting, this must depend on the rate of He migration through the fuzz.

\section{He migration}

We now consider how He continues to reach the $\mathrm{W}$ bulk through the fuzz layer (as isotopic studies showed [36], see Sect. 3.2). Two proposals are explored: diffusion of He down the tendrils, and direct penetration of He through the fuzz.

\subsection{Diffusion}

Fiflis et al. [30] proposed that growth continued by $\mathrm{He}$ bubbles in the bulk loop punching to the surface. He is implanted at the top of the fuzz layer and diffuses down tendrils to the bulk to form bubbles, potentially aided by compressive stress fields of bubbles. Whilst the diffusion of $\mathrm{He}$ in bulk W is much faster than the diffusion coefficients observed, it was proposed that diffusion through the porous fuzz layer would be slower [27].

Klaver et al. [47] listed a series of objections. First, He will diffuse horizontally to tendril edges $(\sim 30 \mathrm{~nm}$ diameter) and desorb well before it manages to diffuse several micrometres vertically to the tendril base. Second, He would become trapped by other interstitial He atoms, bubbles or defects before it reached the bulk. Finally, this diffusion should allow heat conduction, yet fuzz has an extremely low thermal conductivity [7], even when accounting for its high porosity.

A recent MD study [48] placed He atoms in model tendrils, with and without $\mathrm{He}$ bubbles present, to model diffusion. They found that bubbles exerted stresses, driving diffusion as Fiflis et al. [30] proposed. Diffusion coefficients were calculated; these decreased over time as more $\mathrm{He}$ 
clusters form, interacting with the diffusing He. Accounting for desorption at the surface, it was concluded that $\mathrm{He}$ could feasibly diffuse to the base of 1 and $10 \mu \mathrm{m}$ tendrils during formation times. However, this assumed a constant diffusion coefficient for simplicity, despite the findings that coefficients decrease with time. In addition, the possibility of the diffusing $\mathrm{He}$ atoms being absorbed by bubbles was not accounted for.

It appears possible for diffusion to contribute to $\mathrm{He}$ migration; however, further simulations are needed to clarify this. The MD simulations [48] only ran for $20 \mathrm{~ns}$ due to the high computational cost, so coarser methods are needed to extrapolate the findings to longer distances and times. Simulations that could account for He absorption by existing bubbles would also help to identify if diffusion is valid.

\subsection{Ballistic penetration}

Since fuzz layers have been found to have around $90 \%$ porosity, Klaver et al. [47, 49] proposed that $\mathrm{He}^{+}$ions may be able to penetrate much further than the several nanometres expected. They observed that tendrils favour growing perpendicular to the $\mathrm{W}$ surface, leading to open channels that He could scatter down. MD studies showed that $\mathrm{He}$ is able to bounce off fuzz tendrils before implanting, allowing penetrations of the order of micrometres in the most porous fuzz.

Their studies showed an exponential decay in flux with distance into fuzz. This led to the proposal of thickness having logarithmic dependence on fluence or time (fluence $=$ flux $\times$ time) instead of the diffusional square-root dependence seen in Sect. 1:

$x=x_{0} \ln \left(\frac{A}{x_{0}}\left(\Phi-\Phi_{0}\right)+1\right)$.

$x$ : fuzz thickness; $\Phi$ : fluence; $\Phi_{0}$ : incubation fluence; $A$, $x_{0}$ : constants.

The MD calculations were repeated with the computationally simpler binary collision approximation (BCA) approach [49], where ion movement is treated as a sequence of collisions. BCA produced similar results for all but the lowest ion energies, where the approximation is inaccurate. BCA systematically underestimates penetration; however, this can be accounted for, allowing BCA to be used instead of MD calculations taking months. This is significant because a recent BCA study [50] accounted for electronic stopping power (inelastic collisions between ions and bound electrons), which was not included in the original model. The new study reported decreased penetration, but still of several hundred nanometres, indicating penetration remains a viable mechanism when electronic stopping is included.
It should be noted that these simulations must approximate the three-dimensional (3D) structure of fuzz, which has still not been fully determined [50]. TEM observation [51] has shown that tendrils favour growing upwards from the surface; however, they are still interconnected by branches in a complex manner. Figure 5 shows the cylinders used in Ref. [50] to build the structure, whilst [47, 49] used ellipsoids instead. Determination of the complete structure would allow much more accurate modelling to be done, so long as the representations of the structure can be constructed in a way that do not increase computing times.

Further support for ballistic penetration comes from studies using isotopic $\mathrm{He}$ [36]; the ratio of ${ }^{3} \mathrm{He}$ in the sample bulk is similar to the ratio of ${ }^{3} \mathrm{He}$ fluences, which is expected from a direct penetration mechanism. Instead, diffusive $\mathrm{He}$ transport would cause a square-root ratio.

The evidence suggests that ballistic penetration is the primary mechanism for He migration through the fuzz, although it could be aided by diffusion once implanted. We now examine whether penetration is rate-limiting.

\section{Rate-limiting process}

We have established that $\mathrm{W}$ migration is likely due to adatom diffusion, and He migration due to ballistic penetration. If adatom diffusion is rate-limiting, the diffusive (square-root) model should be a good fit to thickness-fluence data. If He penetration (needed to generate adatoms) is limiting, the logarithmic model should apply. Now, we apply these models to experimental data.
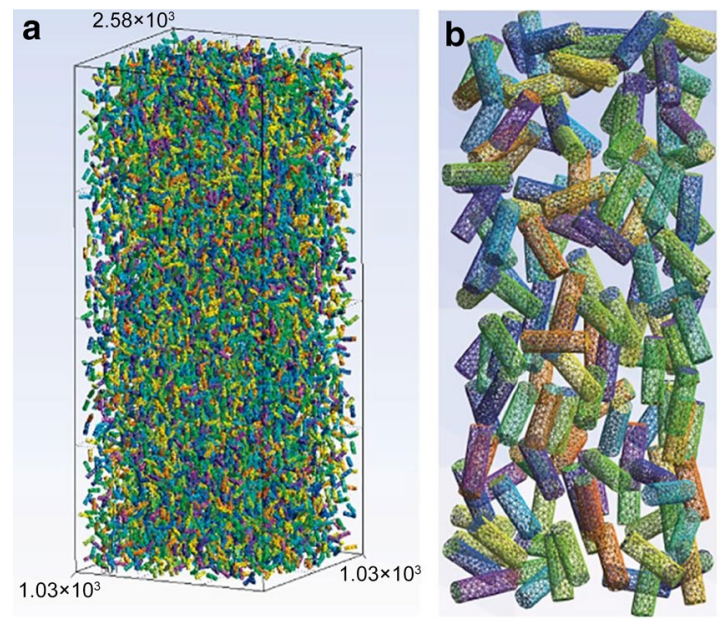

Fig. 5 Fuzz structure used to model He ballistic penetration. a shows the full fuzz structure, with dimensions labelled in nanometres. $\mathbf{b}$ is an enlarged view illustrating the cylinders used to build the fuzz. Reproduced with permission from Ref. [50]. Copyright 2019. Elsevier 


\subsection{Flux dependence}

Rewriting the ballistic relationship in terms of flux gives,

$x=x_{0} \ln \left(\frac{A}{x_{0}}\left(\Gamma t-\Phi_{0}\right)+1\right)$,

so that for constant time, $t$, fuzz thickness, $x$, will increase if there is greater flux, $\Gamma$, allowing more He to reach the fuzz base. The constants and incubation fluence, $\Phi_{0}$, are unaffected.

Instead the diffusional relationship,

$x=\sqrt{\frac{2 D}{\Gamma}\left(\Phi-\Phi_{0}\right)}=\sqrt{2 D\left(t-t_{0}\right)}$,

is independent of flux (using $\Phi=\Gamma t$ ), as adatom diffusion distances only depend upon time. However, there is expected to be a flux threshold below which adatom generation becomes rate-limiting.

Figure 6 shows the variation of thickness with flux. Previously it was concluded that thickness was independent of flux above $\sim 10^{22} \mathrm{~m}^{-2} \cdot \mathrm{s}^{-1}$, as expected for a diffusional mechanism. Here, the ballistic model has been fitted, assuming He migration is limiting across the entire flux range. Due to the large uncertainties in the fluxes, it is difficult to accept or reject the logarithmic fit of all the data.

It is also possible that the ballistic penetration is only limiting below a threshold flux, which will depend on the irradiation conditions. Thus, the logarithmic fit may only apply to data below this threshold. Above this, adatom diffusion becomes limiting and thickness is independent of flux.

\subsection{Fluence dependence}

The two models will be used to see if there is a different relationship between thickness and fluence at different fluxes.

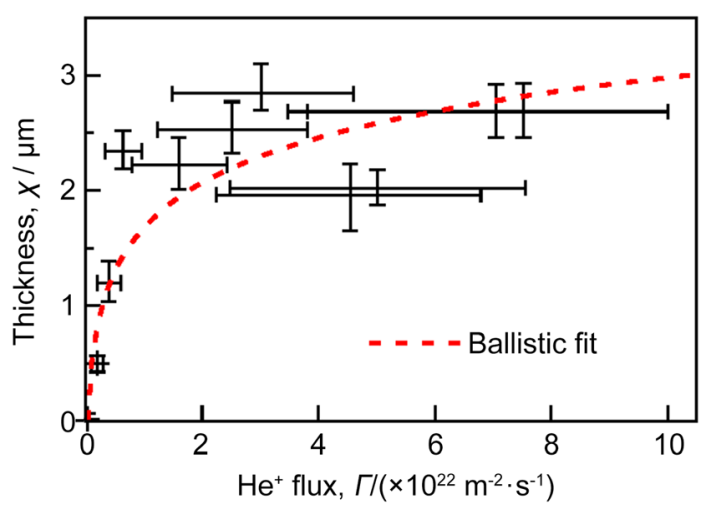

Fig. 6 Variation of fuzz thickness with He flux after $3600 \mathrm{~s}$. The logarithmic dependence proposed by the ballistic penetration model was fitted and shown in red. Data from Ref. [33]
It should be noted that adatom diffusion is independent of the amount of He supplied; however, there is a square-root dependence on fluence simply because fluence is proportional to time.

The data in Fig. 7 were recorded at a flux of $\sim 1.7 \times 10^{22} \mathrm{~m}^{-2} \cdot \mathrm{s}^{-1}$, close to the proposed threshold above which growth is independent of flux. The diffusional model is clearly a worse fit, and predicts a higher incubation fluence ( $x$-intercept) of $6 \times 10^{24} \mathrm{~m}^{-2}$, which is much greater than observed values [21]. At this lower flux, the ballistic model is a much better fit.

The data in Fig. 8 were recorded at a higher flux $\sim 5 \times 10^{22} \mathrm{~m}^{-2} \cdot \mathrm{s}^{-1}$. The ballistic model predicts an unphysical and negative incubation fluence, which also occurred applying the model to similar data [47]. However, applying the ballistic model with $\Phi_{0}=0$ can produce a good fit to the data. The diffusional fit is better than either ballistic fit and has a lower $\chi^{2}$ value (the reduced $\chi^{2}$ is slightly less

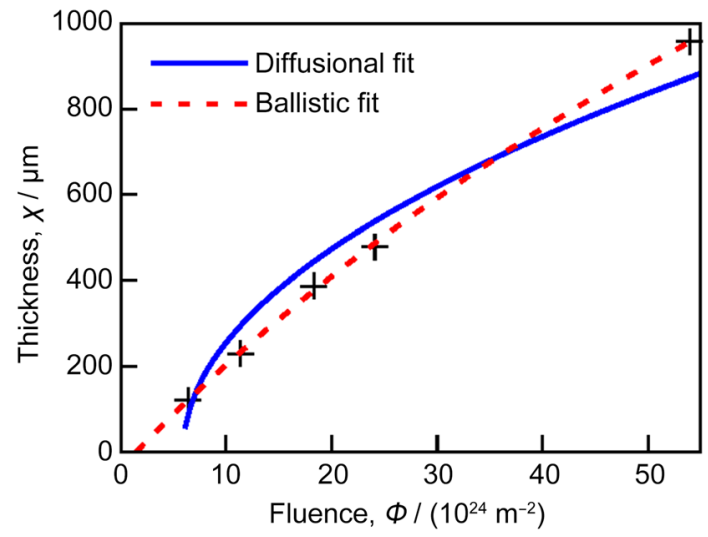

Fig. 7 Fuzz thickness as a function of He fluence. The sample was irradiated with a constant flux of $\sim 1.7 \times 10^{22} \mathrm{~m}^{2} \mathrm{~s}^{-1}$ of $50 \mathrm{eV} \mathrm{He}$ ions at $1400 \mathrm{~K}$. Data from Ref. [14]

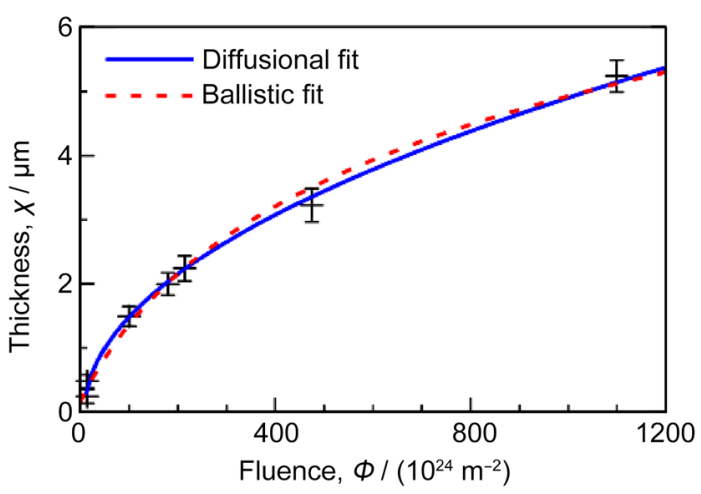

Fig. 8 Fuzz thickness as a function of He fluence. The sample was irradiated with a constant flux of $\sim 5 \times 10^{22} \mathrm{~m}^{2} \mathrm{~s}^{-1}$ of $60 \mathrm{eV} \mathrm{He}^{+}$ions at 1120 K. Data from Ref. [33] 
than 1 but is likely due to an underestimation of errors rather than overfitting). The quantity $\chi^{2}$ is used to evaluate 'goodness of fit' for different models by comparing the residuals to the standard deviation of the original data; a small $\chi^{2}$ implies the model fits the data well. The reduced $\chi^{2}$ accounts for the number of free parameters in a model; ideally this would be close to 1 [52].

Although the ballistic fit at the higher flux cannot be rejected, there is further evidence that diffusion is rate-limiting at higher fluxes. Experiments with identical flux and energy conditions have found that growth rate increases with temperature [27] which is explained by diffusion. Instead, there is no clear reason why the growth rate would increase if ballistic penetration were limiting.

This could imply a flux threshold above which the ratelimiting process switches from $\mathrm{He}$ ballistic penetration to $\mathrm{W}$ adatom diffusion, with the fluence dependence switching from logarithmic to square-root. This would depend upon irradiation conditions, as diffusion will be faster at higher temperatures. Further experiments needed to confirm this are proposed in Sect. 6.

Simulations [16] revealed that the random nature of the loop punching and bubble rupture process, which generates adatoms, could also lead to a $\sqrt{t}$ dependence. However, this also fails to explain the temperature dependence of growth rate.

\section{Conclusion}

Experiments and simulations to clarify the mechanism of late-stage tungsten fuzz growth have been reviewed, looking at helium and tungsten migration in turn. In Sect. 2.3 three questions were presented:

- Is tungsten migration, helium migration, or both responsible for fuzz formation?

- If a helium supply is required, how does it move through the thickening fuzz layer?

- Is tungsten or helium migration rate-limiting?

The answers are summarised below, with areas of debate and further work identified.

Firstly, tungsten adatom migration is likely responsible for growth, shown by the growth rate increase when extra tungsten adatoms are deposited on tendrils [40-42]. However, bubble growth and rupture are needed to form initial protrusions. Adatoms are generated by loop punching as bubbles grow, and can diffuse to protrusion tips to form tendrils. Bubble growth and rupture continue during late-stage growth, and adatoms continue to be generated at the tendril bases.
Second, a constant helium supply to the bottom of the fuzz layer is required for the continued bubble growth and rupture [36]. Ballistic penetration of helium through the porous fuzz layer can explain this, even when electronic stopping is accounted for [50]. However, it appears diffusion could still play a part; for example, diffusion may occur after ballistic penetration. Simulations over longer periods that can account for helium absorption by bubbles during diffusion will help to resolve this.

It would useful to simulate implantation and diffusion together, or to model diffusion starting from the much greater helium implantation distances predicted by ballistic penetration. This requires a better understanding of the fuzz structure, as modelling diffusion through the cylinder structure in Fig. 5 will not be accurate. TEM images of fuzz exist, but Klaver et al. [47] suggest filling the open space with a setting fluid or deposited atoms. This would allow removal of the intact fuzz layer from which TEM topography could identify the complete structure. Using a complete structure determined this way could improve the accuracy of many simulations, not only those related to helium migration but tungsten migration too.

Finally, it is difficult to determine whether helium ballistic penetration or tungsten adatom diffusion is rate-limiting. There is evidence for a flux threshold above which the rate-limiting process switches from helium penetration (logarithmic thickness-fluence relationship) to adatom diffusion (square-root relationship).

High fluence experiments are needed to differentiate accurately between the two models. Repeats at identical temperature and ion energy could investigate if there is a different thickness-fluence relationship at high and low flux. Additional measurements at low helium fluences to determine the incubation fluence could also differentiate between the two models, as the curves fitted in Figs. 7 and 8 have different incubation fluence parameters.

It would also be useful to see if there is a different thickness-fluence relationship at very high fuzz thicknesses if the mechanism changes. Meyer [8] speculates whether adatoms would be generated midway upon tendril sides instead of at the base. To avoid the long times needed for this, tungsten deposition (see Sect. 3.3) could be used to grow a thick fuzz layer, which is then irradiated under normal conditions.

For a phenomenon first observed in 2006 [26], great progress has been made in identifying the mechanism of tungsten fuzz formation. As nuclear fusion enters the DEMOnstration Power Station (DEMO) phase, it will be interesting to see whether fuzz prevents the use of all-tungsten divertors. Understanding the mechanism helps design suppression and removal techniques for reactors, as well as aiding in the production of porous catalysts with high-specific surface areas. 
Acknowledgements The author thanks A. L. Greer for his time and support. This work was produced as part of the author's studies for the MSci in Materials Science at the University of Cambridge.

\section{Declarations}

Conflict of interest The author has no conflicts of interest to declare.

Data availability The data analysed here are available from the original experimental studies referenced.

Open Access This article is licensed under a Creative Commons Attribution 4.0 International License, which permits use, sharing, adaptation, distribution and reproduction in any medium or format, as long as you give appropriate credit to the original author(s) and the source, provide a link to the Creative Commons licence, and indicate if changes were made. The images or other third party material in this article are included in the article's Creative Commons licence, unless indicated otherwise in a credit line to the material. If material is not included in the article's Creative Commons licence and your intended use is not permitted by statutory regulation or exceeds the permitted use, you will need to obtain permission directly from the copyright holder. To view a copy of this licence, visit http://creativecommons.org/licenses/by/4.0/.

\section{References}

1. The beauty of the beast. ITER 2013. http://www.iter.org/newsline/ 252/1449. Accessed 13 Dec 2020

2. Hirai T, Panayotis S, Barabash V, Amzallag C, Escourbiac F, Durocher A, Merola M, Linke J, Loewenhoff Th, Pintsuk G, Wirtz M, Uytdenhouwen I. Use of tungsten material for the ITER divertor. Nucl Mater Energy. 2016;9:616.

3. Ihli T, Hermsmeyer S, Köhly C, Norajitra P. Integration of an advanced He-cooled divertor in a DEMO-relevant tokamak geometry. Fusion Eng Des. 2006;81:121.

4. Kajita S, Sakaguchi W, Ohno N, Yoshida N, Saeki T. Formation process of tungsten nanostructure by the exposure to helium plasma under fusion relevant plasma conditions. Nucl Fusion. 2009;49:095005.

5. Baldwin MJ, Doerner RP. Formation of helium induced nanostructure 'fuzz' on various tungsten grades. J Nucl Mater. 2010;404:165.

6. Temmerman GD, Hirai T, Pitts RA. The influence of plasma-surface interaction on the performance of tungsten at the ITER divertor vertical targets. Plasma Phys Control Fusion. 2018;60:044018.

7. Kajita S, Yagi T, Kobayashi K, Tokitani M, Ohno N. Measurement of heat diffusion across fuzzy tungsten layer. Results Phys. 2016;6:877.

8. Meyer FW. He-ion induced surface morphology change and nanofuzz growth on hot tungsten surfaces. J Phys B At Mol Opt Phys. 2018;52:012001.

9. de Respinis M, Temmerman GD, Tanyeli I, Doerner RP, Baldwin MJ, van de Krol R. Efficient plasma route to nanostructure materials: case study on the use of $\mathrm{m}-\mathrm{WO}_{3}$ for solar water splitting. ACS Appl Mater Interfaces. 2013;5(15):7621-5.

10. Kajita S, Yoshida T, Kitaoka D, Etoh R, Yajima M, Ohno N, Yoshida H, Yoshida N, Terao Y. Helium plasma implantation on metals: Nanostructure formation and visible-light photocatalytic response. J Appl Phys. 2013;113:134301.

11. Hammond KD. Helium, hydrogen, and fuzz in plasma-facing materials. Mater Res Express. 2017;4:104002.
12. Ni WY, Fan HY, Niu CJ, Zhang Y, Liu L, Cui YQ, Dong-Ping $\mathrm{L}$. He bubble-driven growth of $\mathrm{W}$ fuzz during the interaction between $\mathrm{H}_{2} / \mathrm{He}$ plasmas and W materials. Tungsten. 2021;3:393.

13. Budaev VP, Fedorovich SD, Martynenko YuV, Lukashevsky MV, Gubkin MK, Karpov AV, Lazukin AV, Shestakov EA, Kavyrshin DI, Rogozin KS. The PLM plasma device for tests of tungsten with powerful stationary heat plasma loads. Phys At Nucl. 2019;82:1281.

14. Kajita S, Yoshida N, Yoshihara R, Ohno N, Yamagiwa M. TEM observation of the growth process of helium nanobubbles on tungsten: nanostructure formation mechanism. J Nucl Mater. 2011;418:152.

15. Marian J, Becquart CS, Domain C, Dudarev SL, Gilbert MR, Kurtz RJ, Mason DR, Nordlund K, Sand AE, Snead LL, Suzudo $\mathrm{T}$, Wirth BD. Recent advances in modeling and simulation of the exposure and response of tungsten to fusion energy conditions. Nucl Fusion. 2017;57:092008.

16. Lasa A, Tähtinen SK, Nordlund K. Loop punching and bubble rupture causing surface roughening - a model for $\mathrm{W}$ fuzz growth. EPL. 2014;105:25002.

17. Ito AM, Takayama A, Oda Y, Tamura T, Kobayashi R, Hattori T, Ogata S, Ohno N, Kajita S, Yajima M, Noiri Y, Yoshimoto Y, Saito S, Takamura S, Murashima T, Miyamoto M, Nakamura H. Hybrid simulation research on formation mechanism of tungsten nanostructure induced by helium plasma irradiation. J Nucl Mater. 2015;463:109.

18. Ito AM, Takayama A, Nakamura H. Triple hybrid simulation method for tungsten fuzzy nanostructure formation. Plasma Fusion Res. 2018;13:3403061.

19. Sefta F, Juslin N, Wirth BD. Helium bubble bursting in tungsten. J Appl Phys. 2013;114:243518.

20. Fan H, Zhang Y, Liu D, Niu C, Liu L, Ni W, Xia Y, Bi Z, Hong Y, Lei G. Tensile stress-driven cracking of W fuzz over W crystal under fusion-relevant $\mathrm{He}$ ion irradiations. Nucl Fusion. 2020;10:046011.

21. Petty TJ, Baldwin MJ, Hasan MI, Doerner RP, Bradley JW. Tungsten 'fuzz' growth re-examined: the dependence on ion fluence in non-erosive and erosive helium plasma. Nucl Fusion. 2015;55:093033.

22. Miyamoto M, Watanabe T, Nagashima H, Nishijima D, Doerner RP, Krasheninnikov SI, Sagara A, Yoshida N. In situ transmission electron microscope observation of the formation of fuzzy structures on tungsten. Phys Scr. 2014;T159:014028.

23. Wu Y, Liu L, Lu B, Ni W, Liu D. W nano-fuzzes: a metastable state formed due to large-flux $\mathrm{He}^{+}$irradiation at an elevated temperature. J Nucl Mater. 2016;482:294.

24. Hwangbo D, Kawaguchi S, Kajita S, Ohno N. Erosion of nanostructured tungsten by laser ablation, sputtering and arcing. Nucl Mater Energy. 2017;12:386.

25. Noiri Y, Kajita S, Ohno N. Nanostructure growth by helium plasma irradiation to tungsten in sputtering regime. J Nucl Mater. 2015;463:285.

26. Takamura S, Ohno N, Nishijima D, Kajita S. Formation of nanostructured tungsten with arborescent shape due to helium plasma irradiation. Plasma Fusion Res. 2006;1:051.

27. Baldwin MJ, Doerner RP. Helium induced nanoscopic morphology on tungsten under fusion relevant plasma conditions. Nucl Fusion. 2008;48:035001.

28. Petersen NO (2017) Meauring concentration changes. Foundations for nanoscience and nanotechnology. CRC Press, Taylor \& Francis Group, Boca Raton, FL, p 205

29. Karpov VG. Electrostatic theory of metal whiskers. Phys Rev Appl. 2014;1:044001.

30. Fiflis P, Connolly N, Ruzic DN. Experimental mechanistic investigation of the nanostructuring of tungsten with low energy helium plasmas. J Nucl Mater. 2016;482:201. 
31. Woller KB, Whyte DG, Wright GM, Brunner D. Experimental investigation on the effect of surface electric field in the growth of tungsten nano-tendril morphology due to low energy helium irradiation. J Nucl Mater. 2016;481:111.

32. Krasheninnikov SI. Viscoelastic model of tungsten `fuzz' growth. Phys Scr. 2011;T145:014040.

33. Baldwin MJ, Doerner RP, Nishijima D, Tokunaga K, Ueda Y. The effects of high fluence mixed-species (deuterium, helium, beryllium) plasma interactions with tungsten. J Nucl Mater. 2009;390-391:886.

34. Krasheninnikov SI, Smirnov RD. On "bubbly" structures in plasma facing components. J Nucl Mater. 2013;438:S861.

35. Woller KB, Whyte DG, Wright GM. Crystallographic analysis of nano-tendril bundle vs fuzz growth on tungsten exposed to helicon wave-coupled helium plasma. J Appl Phys. 2021;129:115301.

36. Doerner RP, Nishijima D, Krasheninnikov SI, Schwarz-Selinger $\mathrm{T}$, Zach M. Motion of $\mathrm{W}$ and He atoms during formation of $\mathrm{W}$ fuzz. Nucl Fusion. 2018;58:066005.

37. Trufanov D, Marenkov E, Krasheninnikov S. The role of the adatom diffusion in the tungsten fuzz growth. Phys Proced. 2015;71:20.

38. Martynenko YV, Nagel MY. Model of fuzz formation on a tungsten surface. Plasma Phys Rep. 2012;38:996.

39. Yajima M, Yamagiwa M, Kajita S, Ohno N, Tokitani M, Takayama A, Saito S, Ito AM, Nakamura H, Yoshida N. Comparison of damages on tungsten surface exposed to noble gas plasmas. Plasma Sci Technol. 2013;15:282.

40. Kajita S, Kawaguchi S, Ohno N, Yoshida N. Enhanced growth of large-scale nanostructures with metallic ion precipitation in helium plasmas. Sci Rep. 2018;8:56.

41. McCarthy P, Hwangbo D, Bilton M, Kajita S, Bradley JW. Enhanced fuzzy tungsten growth in the presence of tungsten deposition. Nucl Fusion. 2020;60:026012.

42. Kajita S, Yoshida N, Ohno N. Tungsten fuzz: deposition effects and influence to fusion devices. Nucl Mater Energy. 2020;25:100828.
43. Zhang WW, Shi PH, Yao BD, Wu L, Wu XY, Shi LQ, Wang YX. Panorama of "fuzz" growth on tungsten surface under He irradiation. Appl Surf Sci. 2021;542:148543.

44. Patino MI, Nishijima D, Tokitani M, Nagata D, Doerner RP. Material mixing during fuzz formation in W and Mo. Phys Scr. 2020;T171:014070.

45. Patino MI, Nishijima D, Tokitani M, Nagata D, Yu JH, Doerner RP. Material migration in W and Mo during bubble growth and fuzz formation. Nucl Fusion. 2021;61:076001.

46. Sefta F, Hammond KD, Juslin N, Wirth BD. Tungsten surface evolution by helium bubble nucleation, growth and rupture. Nucl Fusion. 2013;53:073015.

47. Klaver TPC, Nordlund K, Morgan TW, Westerhof E, Thijsse BJ, van de Sanden MCM. Molecular dynamics simulations of ballistic He penetration into W fuzz. Nucl Fusion. 2016;56:126015.

48. Cusentino MA, Wirth BD. Helium diffusion and bubble evolution in tungsten nanotendrils. Comput Mater Sci. 2020;183:109875.

49. Klaver TPC, Zhang S, Nordlund K. MD and BCA simulations of $\mathrm{He}$ and $\mathrm{H}$ bombardment of fuzz in bcc elements. J Nucl Mater. 2017;492:113.

50. Yang Z, Song W, Li Y, Yang J. BCA simulations of ion irradiation of tungsten fuzz. Nucl Instrum Methods Phys Res Sect B. 2019;455:118.

51. Wang K, Doerner RP, Baldwin MJ, Meyer FW, Bannister ME, Darbal A, Stroud R, Parish CM. Morphologies of tungsten nanotendrils grown under helium exposure. Sci Rep. 2017;7:42315.

52. Gretarsson AM. Meausrement error. A practical guide to laboratory optics. Cambridge: Cambridge University Press; 2021. p. $37-9$.

Publisher's Note Springer Nature remains neutral with regard to jurisdictional claims in published maps and institutional affiliations. 